\title{
Magnetoelectric effects in superconductors due to spin-orbit scattering: Nonlinear $\sigma$-model description
}

\author{
P. Virtanen $\odot$ \\ Department of Physics and Nanoscience Center, University of Jyväskylä, P.O. Box 35 (YFL), FI-40014 University of Jyväskylä, Finland
}

F. S. Bergeret $\odot$

Centro de Física de Materiales (CFM-MPC) Centro Mixto CSIC-UPV/EHU, E-20018 Donostia-San Sebastián, Spain and Donostia International Physics Center (DIPC), 20018 Donostia-San Sebastián, Spain

I. V. Tokatly $\odot$

Nano-Bio Spectroscopy Group, Departamento de Polímeros y Materiales Avanzados: Física, Química y Tecnología, Universidad del País Vasco (UPV/EHU), 20018 Donostia-San Sebastián, Spain;

IKERBASQUE, Basque Foundation for Science, 48011 Bilbao, Spain;

Donostia International Physics Center (DIPC), 20018 Donostia-San Sebastián, Spain; and ITMO University, Department of Physics and Engineering, 197101 Saint-Petersburg, Russia

(Received 30 June 2021; revised 9 August 2021; accepted 20 August 2021; published 30 August 2021)

\begin{abstract}
We suggest a generalization of the nonlinear $\sigma$ model for diffusive superconducting systems to account for magnetoelectric effects due to spin-orbit scattering. In the leading orders of spin-orbit strength and gradient expansion, it includes two additional terms responsible for the spin-Hall effect and the spin-current swapping. First, assuming a delta-correlated disorder, we derive the terms from the Keldysh path integral representation of the generating functional. Then we argue phenomenologically that they exhaust all invariants allowed in the effective action to the leading order in the spin-orbit coupling (SOC). Finally, the results are confirmed by a direct derivation of the saddle-point (Usadel) equation from the quantum kinetic equations in the presence of randomly distributed impurities with SOC. At this point, we correct a recent derivation of the Usadel equation that includes magnetoelectric effects and does not resort to the Born approximation.
\end{abstract}

DOI: 10.1103/PhysRevB.104.064515

\section{INTRODUCTION}

Spin-orbit coupling (SOC) in solids generates a variety of well-known effects [1-3], such as the spin and the anomalous Hall effects, where magnetic and electric degrees of freedom couple to each other. Two common origins of the effects are often considered, the intrinsic SOC due to properties of the pure lattice, and the extrinsic SOC due to impurities. In superconducting materials, how the different spin-orbital effects manifest, and are conveniently theoretically described, is still partially not resolved.

The magnetoelectric effects associated with SOC due to extrinsic impurity scattering have been extensively discussed in the normal state [1-3], but in the superconducting state have received somewhat less attention [4-6] compared to the intrinsic effects [7]. In contrast, the effect of the spinorbit scattering on spin relaxation in superconductors is well-known [8]. Theoretically, many effects concerning diffusive electron transport in systems with impurities can be described with nonlinear $\sigma$ models [9-12], which also remain convenient when superconductivity is included [12-15]. Spin-orbit relaxation within $\sigma$ models was described early $[10,11]$. However, it is often considered to be generated by a different scattering potential from the normal scattering. This results in an omission of magnetoelectric effects, which to our knowledge were not discussed from this viewpoint.

In this paper, we consider spin-orbit scattering originating from the same potential as the normal scattering, and from this assumption obtain additional terms in the $\sigma$ model, within the simplest expansion in the spin-orbit strength. The result consists of two contributions, corresponding to spin swapping [16] and the spin-Hall effect. The saddle-point equation is similar to the Usadel equation [17] derived in a previous work $[4,6]$. There are, however, certain differences, which we recognize to be due to technical issues in the previous calculations. We explicitly resolve those issues by also rederiving our result from the earlier kinetic equation approach.

The paper is structured as follows. In Sec. II, we derive the Keldysh nonlinear $\sigma$ model including magnetoelectric effects, and discuss its behavior at the saddle point. In Sec. III, we present an alternative derivation of the saddle-point equation, following earlier kinetic equation approaches. Section IV concludes the discussion.

\section{MAGNETOELECTRIC EFFECTS}

We consider superconductors with spin-orbit impurity scattering, described by an action with electron fields on the 
Keldysh contour $C[14,15,18]$,

$$
\begin{aligned}
& S=S_{0}-\int d^{3} r U(\boldsymbol{r}) \int_{C} d t \bar{\Psi}_{\alpha} \hat{V}_{\alpha \beta} \Psi_{\beta}, \\
& \hat{V}=1+i \lambda^{2} \epsilon_{i j k} \sigma_{i} \overleftarrow{\partial}_{r_{j}} \vec{\partial}_{r_{k}} .
\end{aligned}
$$

Here $S_{0}$ is the action without impurities, $U(\boldsymbol{r})$ the disorder potential, and $\lambda$ describes the SOC strength. We have here integrated by parts to move the derivative on $U$ in the SOC term $\boldsymbol{\sigma} \cdot \nabla U \times \boldsymbol{p}$ to act on the field to the left. We introduce the Nambu vectors $\Psi=\left(\psi_{\uparrow}, \psi_{\downarrow}, \bar{\psi}_{\downarrow},-\bar{\psi}_{\uparrow}\right)^{T} / \sqrt{2}$ and $\bar{\Psi}=$ $-i \sigma_{y} \tau_{x} \Psi$ containing the electron fields $\psi$. Moreover, $\tau_{j}=$ $\hat{\sigma}_{j} \otimes 1, \sigma_{j}=1 \otimes \hat{\sigma}_{j}$ are $4 \times 4$ matrices in the Nambu $\otimes$ spin space, composed of $2 \times 2$ Pauli matrices $\hat{\sigma}_{j}$. Summation over the Nambu-spin $\alpha, \beta$ and dimension $i, j, k=x, y, z$ indices is implied, and $\epsilon_{i j k}$ is the antisymmetric tensor. The action $S_{0}$ also contains any source fields, and the superconducting anomalous self-energy matrix $\Delta$ included via standard BCS mean-field decoupling of the interaction. Our formulation here follows Refs. [14,15], and details of the form of the action can be found there, the only difference being the inclusion of SOC in the impurity matrix element shown above.

We now derive a Keldysh $\sigma$-model description of the diffusive transport in this system, including additional terms describing magnetoelectric effects due to the spin-orbit scattering. We first average over the disorder, assuming it is a Gaussian random field with $\left\langle U(\boldsymbol{r}) U\left(\boldsymbol{r}^{\prime}\right)\right\rangle=\frac{1}{2 \pi \nu \tau} \delta\left(\boldsymbol{r}-\boldsymbol{r}^{\prime}\right)$, where $\tau=\tau(\boldsymbol{r})$ is a scattering time (possibly spatially varying) and $v$ the Fermi-level density of states. Gaussian integration yields

$$
\begin{aligned}
S & \mapsto-i \ln \int \mathrm{D}[U] e^{-\pi \nu \tau \int d^{3} r U^{2}(\boldsymbol{r})} e^{i S} \\
& =S_{0}+\int d^{3} r \frac{i}{4 \pi \nu \tau}\left(\int_{C} d t \bar{\Psi}_{\alpha} \hat{V}_{\alpha \beta} \Psi_{\beta}\right)^{2} \\
& =S_{0}+S_{\mathrm{dis}}+S_{1}+S_{2} .
\end{aligned}
$$

The first disorder term $S_{\text {dis }} \propto \lambda^{0}$ contains the quartic disorder interaction [11] independent of the SOC. The averaging also produces additional terms related to spin-orbit scattering, $S_{1} \propto \lambda^{2}$ and $S_{2} \propto \lambda^{4}$. The $\lambda^{4}$ terms lead to spin relaxation and have been previously discussed in the context of $\sigma$ models. [10,11]. However, the $\lambda^{2}$ part, responsible for magnetoelectric effects, is often ignored. This part corresponds to diagrams with connected normal and spin-orbit scattering vertices [19,20], and obtaining them requires considering both on the same footing in the disorder average.

The next step following the typical nonlinear $\sigma$-model scheme is Hubbard-Stratonovich decoupling of the term $S_{\text {dis }} \propto\left[\bar{\Psi}_{\alpha}(\boldsymbol{r}, t) \Psi_{\beta}\left(\boldsymbol{r}, t^{\prime}\right)\right] \times\left[\Psi_{\alpha}(\boldsymbol{r}, t) \bar{\Psi}_{\beta}\left(\boldsymbol{r}, t^{\prime}\right)\right]$ with a local matrix field $Q_{\alpha \beta}\left(\boldsymbol{r}, t, t^{\prime}\right)[10,14,18]$. This leads to an action with residual SOC interaction terms,

$$
S=\frac{i \pi v}{8 \tau} \operatorname{Tr} Q^{2}+\operatorname{Tr} \bar{\Psi}^{T} G^{-1} \Psi+S_{1}+S_{2},
$$

where $G^{-1}=G_{0}^{-1}+\frac{i}{2 \tau} Q$, and $G_{0}$ is the Green function corresponding to $S_{0}$, having the same form as discussed in Ref. [14]. These matrices are $8 \times 8$ size, defined in the Keldysh $\otimes \mathrm{Nambu} \otimes$ spin space. A Keldysh rotation has been applied, transforming the the Keldysh branch index structure to the retarded-advanced block structure $[14,15]$. Here and below, $\mathrm{Tr}$ indicates the matrix trace together with integration over time and position, whereas the matrix trace is denoted by tr.

We are here mainly interested in the magnetoelectric effects, for which it is sufficient to consider SOC perturbatively in the leading order in $\lambda^{2}$. Integrating out fermions to this order leads to

$$
\begin{aligned}
S \mapsto & \frac{i \pi v}{8 \tau} \operatorname{Tr} Q^{2}-\frac{i}{2} \operatorname{Tr} \ln G^{-1}+\frac{i}{4} \operatorname{Tr}\left[\Sigma_{1} G\right], \\
\Sigma_{1}\left(\boldsymbol{r}, \boldsymbol{r}^{\prime}\right)= & \frac{\lambda^{2} \epsilon_{i j k}}{2 \pi i v}\left[\tau\left(\boldsymbol{r}^{\prime}\right)^{-1} \partial_{r_{i}} \delta\left(\boldsymbol{r}-\boldsymbol{r}^{\prime}\right) \sigma_{k} \partial_{r_{j}} G\left(\boldsymbol{r}, \boldsymbol{r}^{\prime}\right)\right. \\
& \left.-\tau(\boldsymbol{r})^{-1} \partial_{r_{i}^{\prime}} \delta\left(\boldsymbol{r}-\boldsymbol{r}^{\prime}\right) \partial_{r_{j}^{\prime}} G\left(\boldsymbol{r}, \boldsymbol{r}^{\prime}\right) \sigma_{k}\right] .
\end{aligned}
$$

Here, $\frac{i}{4} \operatorname{Tr}\left[\Sigma_{1} G\right]=\left\langle S_{1}\right\rangle$ contains the lowest-order self-energy describing the magnetoelectric coupling in the Gaussian disorder model. It has been previously discussed within the quasiclassical theory $[4,6]$.

For a $\sigma$-model action of the above type, the perturbations of the $Q$ matrix are known to contain soft diffusion modes on the manifold $Q^{2}=1$, whereas perturbations that break this condition are massive and suppressed by impurity scattering [9-11]. Hence, following again the typical $\sigma$-model approach, we consider the low-energy action for the modes on the $Q^{2}=$ 1 surface. As in previous works, these can be sought in the form of similarity transforms $Q(\boldsymbol{r})=T(\boldsymbol{r}) \Lambda T(\boldsymbol{r})^{-1}$ describing fluctuations around the saddle point $\Lambda$ of the spatially uniform state, corresponding to $\lambda=0$ but including, e.g., superconductivity [14]. The gradient terms associated with $\lambda \neq 0$ are considered perturbatively on the same footing as those originating from the gradient expansion of the $\operatorname{Tr} \ln$ term. The expansion of the latter in gradients of $T$ and inverse Fermi energy $1 / E_{F}$ is well-known and gives $[10,14,15]$

$$
S_{0}^{\prime}=\frac{i \pi v}{8} \operatorname{Tr}\left[D(\nabla Q)^{2}+4 i \Omega Q\right],
$$

where $D=v_{F} \ell / 3$ is the diffusion constant, $\ell=v_{F} \tau$ the mean-free path, and $\Omega=\epsilon \tau_{3}+\Delta$ contains the time derivative $\epsilon=i \partial_{t} \delta\left(t-t^{\prime}\right)$, the anomalous self-energy matrix $\Delta$, describing superconductivity in the mean-field approximation, and potentially also other local self-energies if they were included in $G_{0}^{-1}$. For simplicity, spherically symmetric dispersion is assumed with Fermi velocity $v_{F}$. We can here also note that the leading SOC contribution beyond Eq. (5), proportional to $\lambda^{4}$, describes the spin relaxation and reads explicitly as follows $[10,11]$ :

$$
S_{2}^{\prime}=\frac{-i \pi v}{8} \operatorname{Tr}\left[\frac{1}{4 \tau_{\mathrm{so}}} Q \sigma_{k} Q \sigma_{k}\right]
$$

where $1 / \tau_{\mathrm{so}}=8 \lambda^{4} p_{F}^{4} / 9 \tau$ is the (Elliot-Yafet) spin relaxation rate (see, e.g., Ref. [4]). Unlike the $\lambda^{2}$ term, this term is also nonzero if SOC and normal scattering are considered to be independent, as was done in Ref. [10].

To evaluate the magnetoelectric $\lambda^{2}$ term in a gradient expansion, we first move to the Wigner representation 


$$
\begin{aligned}
G\left(\boldsymbol{r}_{1}, \boldsymbol{r}_{2}\right) & =\sum_{p} e^{i \boldsymbol{p} \cdot\left(\boldsymbol{r}_{1}-\boldsymbol{r}_{2}\right)} G_{\boldsymbol{p}}\left(\frac{\boldsymbol{r}_{1}+\boldsymbol{r}_{2}}{2}\right), \text { where the term reads } \\
S_{1}^{\prime}= & \frac{i}{4} \operatorname{Tr}\left[\Sigma_{1} G\right]=\int d^{3} r \frac{i \pi \nu \lambda^{2} \epsilon_{i j k}}{4 \tau} \operatorname{tr}\left[i \sigma_{k}\left\langle p_{i} G\right\rangle\left\langle p_{j} G\right\rangle\right. \\
& \left.-\frac{1}{2} \sigma_{k}\left\{\left\langle p_{i} G\right\rangle, \partial_{j}\langle G\rangle\right\}+\frac{i}{4} \sigma_{k} \partial_{i}\langle G\rangle \partial_{j}\langle G\rangle\right],
\end{aligned}
$$

and $\langle f\rangle=\frac{i}{\pi \nu} \sum_{p} f_{p}$. We denote here and below $\partial_{j}=\partial_{r_{j}}$ acting on Wigner transformed functions. To evaluate Eq. (9) up to second order in gradients, the Green's function can be found by solving the Dyson equation

$$
\left(G_{0}^{-1}+\frac{i Q}{2 \tau}\right) G_{p}-\frac{i}{2} \nabla_{p} G_{0}^{-1} \cdot \nabla_{r} G_{p}-\frac{\nabla_{r} Q}{4 \tau} \cdot \nabla_{p} G_{p}=1,
$$

here expanded to first order in gradients, which for this purpose suffices. Iterating the equation once leads to

$$
\begin{gathered}
G_{\boldsymbol{p}} \simeq \mathcal{G}-\frac{i}{2}\left(\mathcal{G} \boldsymbol{v} \cdot \nabla_{r} \mathcal{G}-\nabla_{r} \mathcal{G} \cdot \boldsymbol{v} \mathcal{G}\right), \\
\mathcal{G}(\boldsymbol{r}, \boldsymbol{p})=\frac{1}{G_{0}^{-1}(\boldsymbol{p})+\frac{i}{2 \tau} Q(\boldsymbol{r})}, \quad \boldsymbol{v}=-\nabla_{p} G_{0}^{-1}(\boldsymbol{p}) .
\end{gathered}
$$

The momentum sums can then be evaluated up to accuracy $1 /\left(p_{F} \ell\right)$ :

$$
\langle G\rangle \simeq Q, \quad\left\langle p_{i} G\right\rangle \simeq-\frac{p_{F} \ell}{3} Q \partial_{i} Q .
$$

After substitution of this result into Eq. (9), we find the gradient expansion of the SOC term in leading order,

$$
\begin{aligned}
S_{1}^{\prime} \simeq & \frac{i \pi v}{8} \int d^{3} r\left(-i D \varkappa \epsilon_{i j k} \operatorname{tr}\left[\sigma_{k} \partial_{i} Q \partial_{j} Q\right]\right. \\
& \left.+D \theta \epsilon_{i j k} \operatorname{tr}\left[\sigma_{k} Q \partial_{i} Q \partial_{j} Q\right]\right),
\end{aligned}
$$

where we identify

$$
\varkappa=\frac{2 p_{F}^{2} \lambda^{2}}{3}, \quad \theta=\frac{2 p_{F}^{2} \lambda^{2}}{p_{F} \ell},
$$

as the spin-swapping and the spin-Hall (side-jump) coefficients [16], respectively. Their values agree with Born approximation results for the scattering. The Gaussian disorder assumption precludes obtaining the skew-scattering contribution, but it would only adjust the values of the coefficients, as we discuss below.

The forms of the terms in Eq. (14) can also be argued phenomenologically. First, since $Q^{2}=1$ and $\left\{Q, \partial_{i} Q\right\}=0$, matrix functions $F(Q, \partial Q)$ of second order in derivatives can be expressed as linear combinations of $\partial_{i} Q \partial_{j} Q$ and $Q \partial_{i} Q \partial_{j} Q$. Second, terms of the first order in spin-orbit scattering are also expected to contain traces with one Pauli matrix $\sigma_{k}$. Finally, the invariance under rotations requires that the coefficient tensors are isotropic, and must be proportional to $\epsilon_{i j k}$. Therefore, we are left with only two scalar invariants allowed in the effective action, $\operatorname{Tr}\left[\epsilon_{i j k} \sigma_{k} \partial_{i} Q \partial_{j} Q\right]$ and $\operatorname{Tr}\left[\epsilon_{i j k} \sigma_{k} Q \partial_{i} Q \partial_{j} Q\right]$, which are the forms we have obtained microscopically in Eq. (14). Similarly, one can argue that the spin relaxation term of Eq. (8) is the lowest in gradients (zeroth order) spin-dependent contribution allowed by the time-reversal and rotation invariance.
By combining Eqs. (7), (8), and (14), we obtain the final effective action of the generalized nonlinear $\sigma$ model:

$$
\begin{aligned}
S_{\text {eff }}= & \frac{i \pi v}{8} \operatorname{Tr}\left[D(\nabla Q)^{2}+4 i \Omega Q-\frac{1}{4 \tau_{\mathrm{so}}} Q \sigma_{k} Q \sigma_{k}\right. \\
& \left.-i D \varkappa \epsilon_{i j k} \sigma_{k} \partial_{i} Q \partial_{j} Q+D \theta \epsilon_{i j k} \sigma_{k} Q \partial_{i} Q \partial_{j} Q\right] .
\end{aligned}
$$

This action is the main result of the present paper. It takes into account the main physical effects of extrinsic SOC-the spin relaxation, spin Hall effect, and spin swapping. Importantly, our phenomenological arguments show that only the values of the coefficients may depend on a specific model of disorder, while the form of the action is universal, provided the SOC remains sufficiently weak. In the next section, we will confirm this at the level of the saddle-point equation by deriving it directly from the quantum kinetic equation (KadanoffBaym) equation and going beyond the Born approximation (equivalent to a delta-correlated disorder potential in the path integral).

In Eq. (16), we can recognize that when $D \varkappa$ is spatially constant, the spin-swapping term in Eq. (14) is a total derivative. Hence, only $\nabla_{r}(D \varkappa)$ will appear in the saddle-point equations for $Q$, and its effect on spin accumulation concentrates on, e.g., surfaces where the value of $D \varkappa$ varies. Note also that the spin Hall $\theta$ term we find above is not a total derivative. Without a spin dependence, its counterpart would be $\operatorname{Tr}\left[\epsilon_{i j k} b_{k} Q \partial_{i} Q \partial_{j} Q\right]$, which can exist if the system possess an axial vector $\boldsymbol{b}$. This is a well-known topological term in 2D [21] describing the quantum Hall effect, whereas the spinless counterpart of the swapping term, $\operatorname{Tr}\left[\epsilon_{i j k} b_{k} \partial_{i} Q \partial_{j} Q\right]$, does not appear due to rotation invariance [22].

Let us now include [U(1) and/or $S U(2)]$ vector potential source fields $a_{j}$. In the leading order in the gradient and $1 / p_{F}$ expansions in Eq. (14), they can be added via the covariant replacement $\partial_{j} Q \mapsto \partial_{j} Q-i\left[a_{j}, Q\right]$. The part of $S_{1}^{\prime}$ linear in $a_{j}$ reads

$$
\begin{aligned}
\delta S_{1}^{\prime}= & -\frac{\pi v}{8} \int d^{3} r D \epsilon_{i j k} \operatorname{tr} a_{i}\left[i \varkappa\left[Q \partial_{j} Q, \sigma_{k}\right] Q\right. \\
& \left.+\theta\left\{\partial_{j} Q, \sigma_{k}\right\} Q, Q\right]
\end{aligned}
$$

and provides the contributions to the (spin) current from the SOC. Therefore, the total matrix current takes the following form:

$$
\mathcal{J}_{i}=-D\left(Q \partial_{i} Q-\frac{\epsilon_{i j k}}{4}\left[i \varkappa\left[Q \partial_{j} Q, \sigma_{k}\right] Q+\theta\left\{\partial_{j} Q, \sigma_{k}\right\} Q, Q\right]\right),
$$

where the first term is the usual current originating from the standard action of Eq. (7). Due to the condition $Q^{2}=1$, the result can be expressed in multiple equivalent forms. Here, we have chosen one that simplifies comparisons with the current entering the Usadel equation derived in Refs. [4,6]. For this purpose, it is also instructive to rewrite Eq. (18) in a second 
form:

$$
\begin{aligned}
\mathcal{J}_{i}= & -D\left(Q \partial_{i} Q-i \epsilon_{i j k} \frac{\varkappa}{4}\left[Q \partial_{j} Q, \sigma_{k}+Q \sigma_{k} Q\right]\right. \\
& \left.-\epsilon_{i j k} \frac{\theta}{4}\left\{\partial_{j} Q, \sigma_{k}+Q \sigma_{k} Q\right\}\right) .
\end{aligned}
$$

This representation explains the identification of $\varkappa$ and $\theta$ in Eq. (16) with the swapping coefficient and the spin Hall angle, respectively. In fact, Eq. (4) of Ref. [4] is identical to Eq. (19) up to the replacement $\sigma_{k} \mapsto \frac{1}{2}\left(\sigma_{k}+Q \sigma_{k} Q\right)$, and to Eq. (18) up to a projection $\mathcal{J}_{i} \mapsto \frac{1}{2}\left(\mathcal{J}_{i}-Q \mathcal{J}_{i} Q\right)$. The origin of this difference will be discussed in detail in the next sections.

\section{A. Saddle point}

The saddle-point equation for the action $S_{\text {eff }}$ of Eq. (16) is derived in a usual way [15] by requiring stationarity of the action under the following variation $\delta Q=[w, Q]$, where $w$ is an arbitrary function, which ensures that the condition $Q^{2}=$ 1 is preserved. The result has a form of the Usadel equation $[14,15,17]$, with additional gradient terms originating from the $\lambda^{2}$ part of Eq. (14),

$$
\begin{aligned}
0= & {\left[Q,-i \Omega+\frac{1}{8 \tau_{\mathrm{so}}} \sigma_{k} Q \sigma_{k}+\frac{1}{2} \partial_{k}\left(D \partial_{k} Q\right)\right.} \\
& -\frac{D \theta}{4} \epsilon_{i j k}\left(\partial_{i} Q \partial_{j} Q \sigma_{k}+\partial_{i} Q \sigma_{k} \partial_{j} Q+\sigma_{k} \partial_{i} Q \partial_{j} Q\right) \\
& \left.+i \epsilon_{i j k} \frac{\partial_{i}(D \varkappa)}{4}\left[Q \partial_{j} Q, \sigma_{k}\right] Q+\epsilon_{i j k} \frac{\partial_{i}(D \theta)}{4}\left\{\partial_{j} Q, \sigma_{k}\right\} Q\right],
\end{aligned}
$$

with $Q^{2}=1$. By construction, the equation is of a commutator form, which makes it consistent with the normalization condition. Note that $\left[Q, \frac{1}{2} \partial_{k}\left(D \partial_{k} Q\right)\right]=\partial_{k}\left(D Q \partial_{k} Q\right)$, and that only the derivative of the spin-swapping coefficient $D \varkappa$ enters the equation due to its total derivative form in Eq. (16).

The saddle point (Usadel) Eq. (20) can be rewritten in a physically more transparent form as follows:

$$
[-i \Omega, Q]+\partial_{k} \mathcal{J}_{k}=\mathcal{T}-\frac{1}{8 \tau_{\mathrm{so}}}\left[\sigma_{k} Q \sigma_{k}, Q\right],
$$

where $\mathcal{J}_{k}$ is the matrix current of Eq. (19) [or, equivalently Eq. (18)], and $\mathcal{T}$ a SOC correction to an effective torque originating from the spin Hall and the spin swapping effects $[4,6]$ :

$$
\mathcal{T}=\frac{D}{4} \epsilon_{i j k}\left[\theta\left[\sigma_{k}, Q \partial_{i} Q \partial_{j} Q\right]+i \varkappa\left[\partial_{i} Q \partial_{j} Q, \sigma_{k}\right]\right] .
$$

The saddle point Eq. (21) is identical to the Usadel equations derived in Refs. [4,6] up to one point-an effective renormalization of the spin matrices $\sigma_{k} \mapsto \frac{1}{2}\left(\sigma_{k}+Q \sigma_{k} Q\right)$ in the expression for the current $\mathcal{J}_{k}$ in Eq. (19). As we will see shortly, the reason is an inconsistency in Refs. [4,6] due to neglecting normalization constraints on the perturbative solutions of the Eilenberger equation in the diffusive limit. A corrected calculation recovers the results above. We clarify this issue in the next section.

\section{KINETIC EQUATION DERIVATION}

In this section, we derive the Usadel equation in the presence of SOC from the quantum kinetic equation, which is a more customary way [17,23-26]. We follow here Ref. [6] and restate the main points in the derivation for completeness, up to the point where differences appear.

As in the previous section, we introduce the Keldysh matrix Green's functions (GFs) which is a $8 \times 8$ matrix,

$$
G=\left(\begin{array}{cc}
G^{R} & G^{K} \\
0 & G^{A}
\end{array}\right)
$$

where $G^{R, A, K}$ are the retarded, advanced and Keldysh $4 \times 4$ matrices in the Nambu-spin space. A nontrivial Nambu structure reflects superconducting correlations. In normal systems in the presence of spin-dependent fields [27,28], the Keldysh subblocks are $2 \times 2$ matrices in the spin space. $G$ obeys the equation

$$
\begin{aligned}
& {\left[\tau_{3} i \partial_{t}+\frac{\nabla_{r}^{2}}{2 m}+\mu+\mathbf{h} . \boldsymbol{\sigma}+\Delta-\Sigma\right] G\left(\boldsymbol{r}, t ; \mathbf{r}^{\prime}, t^{\prime}\right)} \\
& \quad=\delta\left(\mathbf{r}-\mathbf{r}^{\prime}\right) \delta\left(t-t^{\prime}\right),
\end{aligned}
$$

where $\mu$ is the chemical potential and $\Delta$ the superconducting order parameter. The self-energy $\Sigma$ describes the impurity scattering, including the SOC term. To obtain the quantum kinetic equation from Eq. (24), one follows a well-known scheme: (i) Subtract from Eq. (24) its conjugate, (ii) perform the Wigner transform, and then (iii) the gradient expansion $[17,23-26]$. Following this procedure, one finally obtains the kinetic equation,

$$
\frac{p_{k}}{m} \partial_{k} G_{\boldsymbol{p}}(\boldsymbol{r})+i \tau_{3} \partial_{t} G_{\boldsymbol{p}}(\boldsymbol{r})-i \partial_{t^{\prime}} G_{\boldsymbol{p}}(\boldsymbol{r}) \tau_{3}=\mathcal{I},
$$

where $\mathcal{I}$ is the collision integral, which is a functional of the Wigner transformed $G_{p}(\boldsymbol{r})$ and $\Sigma_{p}(\boldsymbol{r})$ (see Eq. (18) in Ref. [6]). Because the GFs are peaked at the Fermi level, it is convenient to introduce the quasiclassical GF, which is defined as $\hat{g}(\boldsymbol{n}, \boldsymbol{r})=(i / \pi) \int d \xi G_{\boldsymbol{p}}(\boldsymbol{r})$, where $\boldsymbol{n}$ is a unit vector pointing in the direction of the momentum at the Fermi surface. As in Refs. [4,6], we assume the diffusive limit and expand $\hat{g}$ in spherical harmonics keeping zeroth and first moments, $\hat{g}(\boldsymbol{n}, \boldsymbol{r}) \approx g(\boldsymbol{r})+n_{k} g_{k}(\boldsymbol{r})$. Note that since we consider SOC, generally $\hat{g}(\boldsymbol{n}, \boldsymbol{r})^{2} \neq 1$. The two moments are determined by the following equations [6]:

$$
\tau_{3} \partial_{t} g+\partial_{t^{\prime}} g \tau_{3}+\frac{v_{F}}{3} \partial_{k} g_{k}=\mathcal{I}_{0}, \quad \frac{v_{F}}{3} \partial_{k} g=\mathcal{I}_{k},
$$

where

$$
\begin{aligned}
& \mathcal{I}_{0}\left[g, g_{k}\right]=-i\langle[\Sigma, \mathrm{g}]\rangle-\frac{\partial_{i}}{2}\left\langle\left\{\partial_{p_{i}} \Sigma, \mathrm{g}\right\}\right\rangle, \\
& \mathcal{I}_{k}\left[g, g_{k}\right]=-i\left\langle n_{k}[\Sigma, \mathrm{g}]\right\rangle .
\end{aligned}
$$

Here $\langle\cdot\rangle$ indicates average over momentum direction $\boldsymbol{n}$. These collision integrals have been evaluated in Ref. [6] by expanding the self-energy $\Sigma$ in terms of the small parameter $\lambda^{2} p_{F}^{2}$ up to second order:

$$
\Sigma=\Sigma^{(0)}+\Sigma^{(1)}+\Sigma^{(2)} .
$$

The zeroth order self-energy describes the usual elastic relaxation. The first and second order describe spin-charge 
coupling and the spin relaxation process, respectively $[4,6,19,20]$, similarly as discussed in the previous section. After a lengthy, but straightforward, calculation one can show that Eqs. (26) can be written as [6]

$$
\begin{gathered}
{[-i \Omega, g]+\partial_{k}\left(\frac{v_{F}}{3} g_{k}+\mathcal{J}_{k}^{\mathrm{an}}\right)=\mathcal{T}-\frac{1}{8 \tau_{\mathrm{so}}}\left[\sigma_{a} g \sigma_{a}, g\right],} \\
\frac{v_{F}}{3} \partial_{k} g+\left[\mathcal{A}_{k}, g\right]=0,
\end{gathered}
$$

where $i \Omega=i \epsilon \tau_{3}+i \Delta$ and

$$
\begin{aligned}
\mathcal{J}_{k}^{\text {an }}= & \frac{D}{2} \epsilon_{a k j}\left[\frac{\omega_{1} \tau}{p_{F} l}\left\{\partial_{j} g, \sigma_{a}\right\}+i \frac{\omega_{2} \tau}{p_{F} l}\left[\sigma_{a}, g \partial_{j} g\right]\right], \\
\mathcal{T}= & \frac{D}{4} \epsilon_{a k j}\left[\left(\frac{2}{3} \omega_{2} \tau+\frac{2 \omega_{1} \tau}{p_{F} l}\right)\left[\sigma_{a}, g \partial_{k} g \partial_{j} g\right]\right. \\
& \left.+\left(\frac{2}{3} \omega_{1} \tau-\frac{2 \omega_{2} \tau}{p_{F} l}\right) i\left[\partial_{k} g \partial_{j} g, \sigma_{a}\right]\right]
\end{aligned}
$$

are the matrix anomalous current and spin-orbit torque terms. They are defined in terms of the spin-charge coupling rates $\omega_{1}$ and $\omega_{2}$ which are related to the components of the single-impurity scattering matrix at the Fermi energy: $\hat{t}_{p p^{\prime}}=$ $A+i\left(\boldsymbol{p} \times \boldsymbol{p}^{\prime}\right) \cdot \boldsymbol{\sigma} B / p_{F}^{2}$ via $\omega_{1}=2 \pi n_{\mathrm{im}} N_{F} \operatorname{Re}\left[A^{*} B\right]$ and $\omega_{2}=$ $2 \pi n_{\mathrm{im}} N_{F} \operatorname{Im}\left[A^{*} B\right]$ [6]. Moreover, the matrix $\mathcal{A}_{k}$ in Eq. (31) is defined as

$$
\begin{aligned}
\mathcal{A}_{k}= & \frac{g_{k}}{6 \tau}+\frac{\omega_{1} \epsilon_{a j k}}{6}\left(\frac{1}{3} i\left[g_{j}, \sigma_{a}\right]-\frac{1}{2 p_{F}}\left\{\partial_{j} g, \sigma_{a}\right\}\right) \\
& +\frac{\omega_{2} \epsilon_{a j k}}{12 p_{F}}\left(\sigma_{a} i \partial_{j} g g+g i \partial_{j} g \sigma_{a}\right) \\
& +\frac{\omega_{2} \epsilon_{a j k}}{18}\left(\sigma_{a} g_{j} g-g g_{j} \sigma_{a}\right) .
\end{aligned}
$$

Equations (30) and (31) form a closed system of equations for the zeroth, $g$, and the first moment, $g_{k}$, of the GF. The second equation allows expressing $g_{k}$ in terms of $g$, and after substitution in the first equation, one obtains the Usadel equation. The structure of Eq. (31), ensures on the one hand the normalization condition $g^{2}=1$, which is kept constant by this equation, and on the other hand that $\partial_{k}\left(g \partial_{k} g\right)$ can be represented as a commutator [...,g]. As noted in Refs. [4,6], even though in the kinetic equation approach the normalization condition is not assumed from the outset, it reappears in the diffusive limit, reflecting a similar structure in the $\sigma$ model. In other words, independently of the way used to derive it, the Usadel equation must have the commutator structure

$$
[\Lambda, g]=0,
$$

where $\Lambda$ is a certain matrix.

Consequently, one can now suspect that the Usadel equation derived in Ref. [6] is not correct, because it does not preserve the commutator form. The cause of this inconsistency is in the procedure for solving the equation system Eqs. (30) and (31). This can be corrected to obtain a consistent solution, as we discuss next.

The procedure is to express $g_{k}$ in terms of $g$ using Eq. (31), and then substitute this expression into Eq. (30). We work in the leading order in small SOC and write the first moment as

$$
g_{k}=-\lg \partial_{k} g+\delta g_{k},
$$

where $\delta g_{k}$ is the correction due to SOC. From Eq. (31), we get the equation for $\delta g_{k}$,

$$
\left[g, \frac{\delta g_{k}}{6 \tau}-\frac{v_{F} \epsilon_{a k j}}{12}\left(\theta^{\prime}\left\{\partial_{j} g, \sigma_{a}\right\}+i \varkappa^{\prime}\left[g \partial_{j} g, \sigma_{a}\right]\right)\right]=0,
$$

where the parameters $\theta^{\prime}$ and $\varkappa^{\prime}$ are defined as follows:

$$
\varkappa^{\prime}=\frac{2}{3} \omega_{1} \tau-\frac{\omega_{2} \tau}{p_{F} l}, \quad \theta^{\prime}=\frac{2}{3} \omega_{2} \tau+\frac{\omega_{1} \tau}{p_{F} l},
$$

The commutator Eq. (37) has multiple solutions. In Ref. [6], the authors choose the solution for $\delta g_{k}$ which nullifies the second term in the commutator. It is this choice that in the end leads to an equation which does not have the commutator structure, and hence does not ensure the normalization condition. To obtain the correct Usadel equation, one can note that the general solution of Eq. (37) can be written as [29]

$$
\delta g_{k}=\frac{l \epsilon_{a k j}}{2}\left(\theta^{\prime}\left\{\partial_{j} g, \sigma_{a}\right\}+i \varkappa^{\prime}\left[g \partial_{j} g, \sigma_{a}\right]\right)+\left\{\Gamma_{k}, g\right\},
$$

where $\Gamma_{k}$ has to be determined by imposing, that after substitution in Eq. (30), one obtains the Usadel equation with the form of Eq. (35).

To find the value of $\Gamma_{k}$, let us focus on the derivative term on the left-hand side of Eq. (30). It is a total divergence that defines, after substitution of Eqs. (32), (36), and (39), the total current $\mathcal{J}_{k}=\frac{v_{F}}{3} g_{k}+\mathcal{J}_{k}^{\text {an }}$ :

$$
\begin{aligned}
\mathcal{J}_{k}= & -D g \partial_{k} g+\frac{D \epsilon_{a k j}}{2} \theta\left\{\partial_{j} g, \sigma_{a}\right\} \\
& +\frac{D \epsilon_{a k j}}{2} i \varkappa\left[g \partial_{j} g, \sigma_{a}\right]+\frac{v_{F}}{3}\left\{\Gamma_{k}, g\right\},
\end{aligned}
$$

where $\theta \equiv \theta^{\prime}+\frac{\omega_{1} \tau}{p_{F} l}=\frac{2}{3} \omega_{2} \tau+2 \frac{\omega_{1} \tau}{p_{F} l}$ and $\varkappa \equiv \varkappa^{\prime}+\frac{\omega_{2} \tau}{p_{F} l}=$ $\frac{2}{3} \omega_{1} \tau-2 \frac{\omega_{2} \tau}{p_{F} l}$ are the spin Hall angle and spin swapping coefficient, which reduce to Eqs. (15) in the Born approximation.

The derivative term in Eq. (30) now has a form $\partial_{k}\left(D \theta A_{k}+\right.$ $\left.D \varkappa B_{k}+\left(v_{F} / 3\right)\left\{\Gamma_{k}, g\right\}\right)$. Notice that the derivative acts also on the kinetic coefficients. The matrix $\Gamma_{k}$ is then obtained by imposing that such terms $\propto \partial_{k}(D \kappa), \partial_{k}(D \theta)$ have the commutator form, Eq. (35) [30]:

$$
\Gamma_{k}=-\frac{l \epsilon_{a k j}}{4} \theta\left\{\partial_{j} g, \sigma_{a}\right\} g-\frac{l \epsilon_{a k j}}{4} i \varkappa\left[g \partial_{j} g, \sigma_{a}\right] g .
$$

Thus, finally we we can write the expression for the current as

$$
\mathcal{J}_{k}=-D g \partial_{k} g+\frac{D \epsilon_{a k j}}{4}\left[\theta\left\{\partial_{j} g, \sigma_{a}\right\} g+i \varkappa\left[g \partial_{j} g, \sigma_{a}\right] g, g\right] .
$$

This result is identical to Eq. (18) and therefore the form of Eq. (19) with the renormalized spin matrices. We can note that the resulting spin-orbit terms in Eq. (42) are exactly those generated by Eq. (17), and originate from the covariant derivatives in the $\sigma$-model action.

With the form of $\mathcal{J}_{k}$ now fixed, the Usadel equation reads

$$
[-i \Omega, g]+\partial_{k} \mathcal{J}_{k}=\mathcal{T}-\frac{1}{8 \tau_{\mathrm{so}}}\left[\sigma_{a} g \sigma_{a}, g\right],
$$


and exactly coincides with the saddle point Eq. (21). From here, we can already conclude that this equation can be expressed in a commutator form. It is, however, instructive to check this directly, and indeed using antisymmetry of $\epsilon_{i j k}$ and the normalization condition $g^{2}=1$, one straightforwardly brings the equation to the form

$$
\begin{aligned}
0= & {\left[g,-i \Omega+\frac{1}{8 \tau_{\mathrm{so}}} \sigma_{k} g \sigma_{k}+\partial_{k} \frac{D}{2} \partial_{k} g\right.} \\
& +\epsilon_{a k j} \frac{D \theta}{4}\left(\partial_{j} g \sigma_{a} \partial_{k} g+\sigma_{a} \partial_{j} g \partial_{k} g+\partial_{j} g \partial_{k} g \sigma_{a}\right) \\
& \left.+\epsilon_{a k j} \frac{\partial_{k}(D \theta)}{4}\left\{\partial_{j} g, \sigma_{a}\right\} g+i \epsilon_{a k j} \frac{\partial_{k}(D \varkappa)}{4}\left[g \partial_{j} g, \sigma_{a}\right] g\right] .
\end{aligned}
$$

This result coincides with the commutator form Eq. (20) of the saddle-point equation for the $\sigma$ model defined by Eq. (16). It is worth emphasizing that in the kinetic derivation of the present section, we have treated the scattering of a single impurity exactly [6], far beyond the model of $\delta$-correlated disorder adopted in Sec. II. This only leads to changes of the coefficients, whereas the structure of the saddle point equation, Eq. (21), and hence of the underlying $\sigma$ model, remains unchanged, in agreement with our symmetry-based arguments in Sec. II.

\section{CONCLUSIONS}

We have derived terms originating from spin-orbit impurity scattering in the Keldysh nonlinear $\sigma$-model action for superconducting systems, which are the source of magnetoelectric effects. The saddle-point equation of the resulting action is the Usadel equation, Eq. (21), which includes effects proportional to the spin swapping coefficient and the spin-Hall angle. We have also discussed a way to derive the Usadel equation via the kinetic equation approach, noting corrections to previously obtained results. Our findings provide a general approach for describing magnetoelectric effects due to extrinsic spin-orbit scattering in diffusive superconductors, both in and out of equilibrium. The approach is also amenable for considering fluctuation effects, away from the saddle point, in systems with SOC.

\section{ACKNOWLEDGMENTS}

P.V. and F.S.B. acknowledge funding from EU's Horizon 2020 research and innovation program under Grant Agreement No. 800923 (SUPERTED). P.V. acknowledges funding from Academy of Finland Project 317118. I.V.T. acknowledges support by Grupos Consolidados UPV/EHU del Gobierno Vasco (Grant No. IT1249-19). F.S.B. acknowledges funding by the Spanish Ministerio de Ciencia, Innovacion y Universidades (MICINN) (Project No. FIS2017-82804-P).
[1] I. Žutić, J. Fabian, and S. Das Sarma, Spintronics: Fundamentals and applications, Rev. Mod. Phys. 76, 323 (2004).

[2] N. Nagaosa, J. Sinova, S. Onoda, A. H. MacDonald, and N. P. Ong, Anomalous Hall effect, Rev. Mod. Phys. 82, 1539 (2010).

[3] J. Sinova, S. O. Valenzuela, J. Wunderlich, C. H. Back, and T. Jungwirth, Spin Hall effects, Rev. Mod. Phys. 87, 1213 (2015).

[4] F. S. Bergeret and I. V. Tokatly, Manifestation of extrinsic spin Hall effect in superconducting structures: Nondissipative magnetoelectric effects, Phys. Rev. B 94, 180502(R) (2016).

[5] C. Espedal, P. Lange, S. Sadjina, A. G. Mal'shukov, and A. Brataas, Spin Hall effect and spin swapping in diffusive superconductors, Phys. Rev. B 95, 054509 (2017).

[6] C. Huang, I. V. Tokatly, and F. S. Bergeret, Extrinsic spincharge coupling in diffusive superconducting systems, Phys. Rev. B 98, 144515 (2018).

[7] V. M. Edelstein, Magnetoelectric Effect in Polar Superconductors, Phys. Rev. Lett. 75, 2004 (1995).

[8] A. A. Abrikosov and L. P. Gor'kov, Spin-orbit interaction and the Knight shift in superconductors, Zh. Eksp. Teor. Fiz. 42, 1088 (1962) [JETP 15, 752 (1962)].

[9] F. Wegner, The mobility edge problem: Continuous symmetry and a conjecture, Z. Phys. B 35, 207 (1979).

[10] K. Efetov, A. Larkin, and D. Kheml'nitskii, Interaction of diffusion modes in the theory of localization, Zh. Eksp. Teor. Fiz. 79, 1120 (1980) [JETP 52, 568 (1980)]

[11] K. B. Efetov, Supersymmetry and theory of disordered metals, Adv. Phys. 32, 53 (1983).

[12] D. Belitz and T. R. Kirkpatrick, The Anderson-Mott transition, Rev. Mod. Phys. 66, 261 (1994).
[13] A. M. Finkel'shtein, Superconducting transition temperature in amorphous films, JETP Lett. 45, 46 (1987).

[14] M. V. Feigel'man, A. I. Larkin, and M. A. Skvortsov, Keldysh action for disordered superconductors, Phys. Rev. B 61, 12361 (2000).

[15] A. Kamenev and A. Levchenko, Keldysh technique and nonlinear $\sigma$-model: Basic principles and applications, Adv. Phys. 58, 197 (2009).

[16] M. B. Lifshits and M. I. Dyakonov, Swapping Spin Currents: Interchanging Spin and Flow Directions, Phys. Rev. Lett. 103, 186601 (2009).

[17] K. D. Usadel, Generalized Diffusion Equation for Superconducting Alloys, Phys. Rev. Lett. 25, 507 (1970).

[18] A. Kamenev, Field Theory of Non-Equilibrium Systems (Cambridge University Press, Cambridge, UK, 2011).

[19] R. Raimondi and P. Schwab, Interplay of intrinsic and extrinsic mechanisms to the spin Hall effect in a two-dimensional electron gas, Physica E 42, 952 (2010).

[20] K. Shen, R. Raimondi, and G. Vignale, Theory of coupled spincharge transport due to spin-orbit interaction in inhomogeneous two-dimensional electron liquids, Phys. Rev. B 90, 245302 (2014).

[21] H. Levine, S. B. Libby, and A. M. M. Pruisken, Electron Delocalization by a Magnetic Field in Two Dimensions, Phys. Rev. Lett. 51, 1915 (1983).

[22] A. Altland and B. Simons, Condensed Matter Field Theory (Cambridge University Press, Cambridge, UK, 2010).

[23] G. Eilenberger, Transformation of Gorkov's equation for type II superconductors into transport-like equations, Z. Phys. 214, 195 (1968). 
[24] G. M. Eliashberg, Inelastic electron collisions and nonequilibrium stationary states in superconductors, JETP 34, 668 (1972) [Zh. Eksp. Teor. Fiz. 61, 1254 (1971)].

[25] A. Schmid and G. Schön, Linearized kinetic equations and relaxation processes of a superconductor near $t_{c}$, J. Low Temp. Phys. 20, 207 (1975).

[26] A. I. Larkin and Y. N. Ovchinnikov, in Nonequilibrium Superconductivity, edited by D. N. Langenberg and A. I. Larkin (Elsevier, Amsterdam, 1986), p. 493.

[27] C. Gorini, P. Schwab, R. Raimondi, and A. L. Shelankov, NonAbelian gauge fields in the gradient expansion: Generalized
Boltzmann and Eilenberger equations, Phys. Rev. B 82, 195316 (2010).

[28] K. Morawetz, Kinetic theory of spin-polarized systems in electric and magnetic fields with spin-orbit coupling. I. Kinetic equation and anomalous Hall and spin-Hall effects, Phys. Rev. B 92, 245425 (2015).

[29] To see that this is the general solution, one can note that $[g, X]=0$ obtains the simple form $\left[\tau_{3}, \tilde{X}\right]=0$ in the eigenbasis of $g$, after which the statement follows.

[30] In general, if $g^{2}=1$ and one searches for a matrix $\Gamma$ such that $A+\{\Gamma, g\}$ has a commutator form, then $\Gamma=-(1 / 2) A g$. 